\title{
Development of biochemical methods to estimate the subcellular impact of uranium exposure on Chlamydomonas reinhardtii
}

\author{
C. Pradines, V. Wiktor, V. Camilleri and R. Gilbin \\ Laboratory of Radioecology and Ecotoxicology, Institute for Radioprotection and Nuclear \\ Safety, DEI/SECRE/LRE, Cadarache, Bd. 186, BP. 3, \\ 13115 St-Paul-lez-Durance Cedex, France
}

\begin{abstract}
This work aims at determining early effects of uranium on the green microalga Chlamydomonas reinhardtii. First, the global effect on growth rate inhibition of exponentially-growing cultures was assessed on favourable conditions for uranium bioavailability (e.g. $\mathrm{pH}=5$ ), $\mathrm{EC}_{50}-24 \mathrm{hrs}$ equals roughly to $150 \mathrm{nM}$ whatever the uranium isotopic composition considered (depleted $\mathrm{U}$ or ${ }^{233} \mathrm{U}$ ). Then, the sensitivity of different parameters representative of (i) oxidative stress (GSH/[GSH +0.5 GSSG] ratio) (ii) metal detoxifying (phytochelatins production) and (iii) photosynthetic activity (chlorophyll fluorescence) was tested. Setting assay of different forms of glutathione and phytochelatins by HPLC was firstly optimised with cadmium-contaminated cells. This assay completed by chlorophyll fluorescence and algal growth was subsequently applied on samples contaminated by $150 \mathrm{nM}$ of depleted uranium or ${ }^{233} \mathrm{U}$. No phytochelatin was produced in our experimental conditions. No difference of $\mathrm{GSH} /[\mathrm{GSH}+0.5 \mathrm{GSSG}]$ ratio was shown between control and contaminated algae. This result suggests that the algae could be stressed before contamination due to culture condition. Chlorophyll fluorescence measurement showed photosynthetic activity inhibition after $24 \mathrm{hrs}$, in the same way for depleted uranium and ${ }^{233} \mathrm{U}$. Thus, the effect observed on the photosynthetic activity could be mainly attributed to the chemical toxicity of the metal.
\end{abstract}

\section{INTRODUCTION}

Uranium range of concentration in freshwater environments is conditioned by the composition, $\mathrm{pH}$ and redox potential of the media [1]. Currently available ecotoxicological data on uranium in aquatic organisms concern essentially acute exposure and few data deal with microalgae. Nevertheless, recent studies have shown that uranium toxicity on Chlorella sp. is highly dependent on $\mathrm{pH}$ and hardness [2-3]. Moreover, the bioavailability of uranium towards $C$. reinhardtii was recently investigated and the authors showed that metal short-term uptake was reduced by inorganic ligands such as phosphate, and organic ligands such as citrate and ethylenediaminetetraacetic acid [4]. To our knowledge, there is no data concerning uranium subcellular toxicity on C. reinhardtii. However, the induction of subcellular damage is the first earlier measurable signal for potential toxicity of uranium. These toxic effects could be related to chemical and/or radiological properties of the isotope. Uranium, such numerous xenobiotics, is able to promote oxidative stress [1]. This phenomenon is commonly observed on aquatic organisms in response to chemical (organic and inorganic pollutant) and/or physical pollution (temperature, radiation, etc.). Furthermore, it was proved that algae respond to heavy metal by induction of antioxidant enzymes and synthesis of low molecular weight compounds such as glutathione [5]. Phytoplankton was also shown to produce small metal-binding proteins (phytochelatins) in response of various metals accumulation in the cytosol [6]. The function of 
phytochelatins consists in chelating free metal in order to decrease its cytosolic concentration. At present, there is no data relating to the ability of phytochelatins to complex uranium and/or to be induced by this metal. In order to characterize the impact of uranium contamination on C. reinhardtii, we propose to investigate different parameters related to global metabolism, oxidative stress and metal detoxification. The objectives of this work were to characterize the processes implied in uranium toxicity at the cellular level, in conditions where uranium was shown to inhibit the growth rate of exponentially-growing populations of algae. The experiments were realised with depleted $\mathrm{U}$ and ${ }^{233} \mathrm{U}$ with the aim to investigate potential differences between chemical and radiological toxicity of the metal. Algae were firstly exposed to different concentrations of depleted uranium in order to define the concentration, which inhibits $50 \%$ of cells growth $\left(\mathrm{EC}_{50}-24,-48\right.$ and $\left.-72 \mathrm{hrs}\right)$. This concentration was used in a second exposure assay during which C. reinhardtii were exposed to depleted $U$ and ${ }^{233} U$. The evolution of different biomarkers, representative of general metabolism (growth rate, photosynthesis), oxidative stress (glutathione redox ratio), metal detoxification (phytochelatin production), was analysed throughout the first $24 \mathrm{hrs}$ of exposure for all treatments conditions.

\section{MATERIAL AND METHODS}

\subsection{Experimental procedure}

The experiments were carried out with the green alga C. reinhardtii (from CCAP, Cumbria, United Kingdom; culture 11/32B) in defined inorganic media. Cells were grown axenically in a medium with reduced nitrate and phosphate concentrations (HSM-U) in order to avoid uranium complexation. The composition of HSM-U [7] compared to original HSM media [8] is presented in table 1. Asynchronous and axenic cultures of exponentially-growing cells were maintained at $24 \pm 2^{\circ} \mathrm{C}$, continuous illumination $\left(100 \pm 10 \mu \mathrm{mol} \cdot \mathrm{m}^{-2} \cdot \mathrm{s}^{-1}\right)$, agitation $(130 \mathrm{rpm})$, and $\mathrm{pH}(5$ and 7 for contamination and stock media, respectively). All plastic ware and glassware were soaked for at least $24 \mathrm{hrs}$ in $2 \% \mathrm{w} / \mathrm{v} \mathrm{HNO}_{3}$, thoroughly rinsed seven times with ultrapure water $\left(18 \mathrm{M} \Omega . \mathrm{cm}^{-1}\right)$ and sterilised by autoclaving prior to use. All the transfers of sterile media and axenic algae were performed under sterile conditions, in a class 100 laminar flow hood.

Table 1. Molar concentrations of major cations and anions in the standard HSM medium and the modified medium used in this study (HSM-U).

\begin{tabular}{|c|c|c|c|c|c|c|c|c|c|}
\hline Ions & $\mathrm{Ca}$ & $\mathrm{Mg}$ & $\mathrm{Na}$ & K & $\mathrm{Cl}$ & $\mathrm{SO}_{4}$ & $\mathrm{NO}_{3}$ & $\mathrm{NH}_{4}$ & $\mathrm{PO}_{4}$ \\
\hline HSM & $6.8010^{-5}$ & $8.1210^{-5}$ & $1.0210^{-4}$ & $2.2010^{-2} 9$ & $9.4910^{-3} 8$ & $8.1210^{-5}$ & - & $9.3510^{-3}$ & $1.3710^{-2}$ \\
\hline HSM-U & $6.8010^{-5}$ & $8.1210^{-5}$ & $1.0210^{-4}$ & $8.2210^{-3} 5$ & $5.9810^{-6}$ & $8.1210^{-5} \mathrm{~g}$ & $9.0710^{-3}$ & $9.3710^{-4}$ & $1.3710^{-4}$ \\
\hline
\end{tabular}

Algae were acclimated in media at $\mathrm{pH} 5$ prior tests. The same experiments were performed with depleted U (uranyl nitrate, Sigma, France) and ${ }^{233} \mathrm{U}$ (CERCA-LEA Framatome, France). For growth inhibition experiments, cells were inoculated at 2500 cell. $\mathrm{mL}^{-1}$ in $100 \mathrm{~mL}$ of sterile HSM-U medium at $\mathrm{pH} 5$ in $250 \mathrm{~mL}$ polycarbonate erlenmayers. Samplings and $\mathrm{pH}$ adjustment were performed at 24,48 and $72 \mathrm{hrs}$. Experiments were performed in triplicate, with a control condition and 5 uranium concentrations from 50 to $350 \mathrm{nmol} \mathrm{U.L^{-1 }}$. For subcellular effects experiments, cells were initially inoculated at ca. 500000 cell. $\mathrm{mL}^{-1}$ in triplicate and sample aliquots taken after 3, 6, 12 and $24 \mathrm{hrs}$. Cadmium and uranium contamination experiments were carried out with respectively $10 \mu \mathrm{M}$ and $150 \mathrm{nM}$ of metal. Algal density and biovolume were measured using Z2 Coulter counter (Beckman Coulter, France). Concentration-effect curves were fitted with Regtox macro for Excel ${ }^{\mathrm{TM}}$ (http://perso.wanadoo.fr/eric.vindimian/) by the Hill model and the $\mathrm{EC}_{50} \mathrm{~S}$ (effective concentration leading to a $50 \%$ decrease of the growth-rate) were calculated with their $95 \%$ confidence interval. 
All exposure concentrations in the media were checked at the beginning and at the end of the experiments. ${ }^{233} \mathrm{U}$ activities were measured by liquid scintillation (Quantulus 1220, Wallac Oy, Turku, Finland; detection limit: $30 \mathrm{mBq}$ ). U depl. concentrations were measured by ICP-AES (Optima 4300 DV, Perkin Elmer). Concentrations were checked to vary by less than $10 \%$ during experiments. Results are expressed according to the initial concentration measurements in the media tested.

\subsection{Sampling and analysis of subcellular parameters}

At the end of exposure cells were harvested by centrifugation $\left(18500 \mathrm{x} \mathrm{g}, 15 \mathrm{~min}, 20^{\circ} \mathrm{C}\right)$ and the pellets resuspended in adapted volume of extraction buffer $(6,3 \mathrm{mM}$ DTPA - $0,1 \%$ TFA $)$ to reach about $60 \times 10^{6}$ cells. $\mathrm{mL}^{-1}$. Samples aliquots were stored at $-80^{\circ} \mathrm{C}$ until glutathione and PCs analysis. Before analysis, sample aliquots were disrupted by sonication. The homogenates were centrifuged at $18500 \times 5$ for $15 \mathrm{~min}$ at $4^{\circ} \mathrm{C}$. The analyses of non-protein thiols compounds were carried out by HPLC using pre-column labeling methods modified for unicellular algae [9]. $125 \mu \mathrm{L}$ of supernatant were mixed with $225 \mu \mathrm{L}$ of reaction buffer [0.2 M 4-(2-hydroxy-ethyl)-piperazine-1-propane-sulfonic acid $\mathrm{pH} 8.2$ containing $6.3 \mathrm{mM}$ DTPA] and $5 \mu \mathrm{L}$ of $25 \mathrm{mM}$ monobromobimane (mBBr) dissolved in acetonitrile. Following $30 \mathrm{~min}$ of incubation in the dark at room temperature (allowing $\mathrm{mBBr}$ to complex with the thiols), the reaction was stopped by adding $150 \mu \mathrm{L}$ of $1 \mathrm{M}$ methane sulfonic acid. The samples were stored at $4^{\circ} \mathrm{C}$ in the dark until analysis. The thiol concentrations in the extract were converted in nmol SH. L ${ }^{-1}$. The reduction step consisted of mixing $125 \mu \mathrm{L}$ of supernatant with $215 \mu \mathrm{L}$ of reaction buffer and $10 \mu \mathrm{L}$ of $10 \mathrm{mM}$ Tris (2-carboxyethyl) phosphine (TCEP). After $20 \mathrm{~min}$ at room temperature, $5 \mu \mathrm{L}$ of $50 \mathrm{mM} \mathrm{mBBr}$ were added to the reaction mixture and incubated in the dark, at room temperature for $30 \mathrm{~min}$. the reaction was stopped by adding $150 \mu \mathrm{L}$ of $1 \mathrm{M}$ methane sulfonic acid and the samples were stored at $4^{\circ} \mathrm{C}$ in the dark. The bimane derivatives were separated on reverse-phase Nova-Pak C18 analytical column (pore size, $60 \AA$; particle size, $4 \mu \mathrm{M}$; dimensions, 3.9 by $300 \mathrm{~mm}$; Waters catalog no. 11695) using gradient of $0.08 \%(\mathrm{v} / \mathrm{v})$ TFA/acetonitrile in $0.1 \%(\mathrm{v} / \mathrm{v})$ $\mathrm{TFA} / \mathrm{H}_{2} \mathrm{O}$ at a flow rate of $1 \mathrm{~mL}$. $\mathrm{min}^{-1}$. Fluorescence $(380 / 470 \mathrm{~nm})$ was monitored by a fluorescence detector (RF 2000, DIONEX). Calibration curves of glutathione were used in all measurements. Cysteine, GSH (both from Sigma) and three PCs ( $=2,3$ and 4) chemically synthesized were used as standards. For the fluorescence measurement $100 \mu \mathrm{L}$ of culture were mixed with equal volume of HSM-U with or without $2 \mu$ of DCMU. The fluorescence $(450 / 680 \mathrm{~nm})$ was measured using microplate specrofluorometer (SpectraMax GEMINI XS, molecular devices).

\section{RESULTS}

\subsection{Growth inhibition results}

The $\mathrm{EC}_{50}$ of $\mathrm{U}$ depl. was found to be close to 150 nmol U.L $\mathrm{L}^{-1}$ whatever the time considered (table 2). No additional toxicity due to the radiation of ${ }^{233} \mathrm{U}$ was observed below $48 \mathrm{~h}$, compared to the growth inhibition observed with $U$ depl. Toxic effect decreased with ${ }^{233} \mathrm{U}$ after $72 \mathrm{hrs}$. As a consequence, it was decided to expose cells to $150 \mathrm{nM}$ on a short-term period (up to $24 \mathrm{~h}$ ) to check subcellular effects. As the quantities of algae need for the analysis is high, exposure was performed at high algae density (ca. $500000 \mathrm{cell}^{\mathrm{mL}} \mathrm{mL}^{-1}$ ).

Table 2. Toxicity of $\mathrm{U}$ depl. and ${ }^{233} \mathrm{U}$ on the growth rate of $C$. reinhardtii in HSM-U, pH 5 (optimal estimate with $95 \%$ confidence interval).

EC50 nmol U.L ${ }^{-1}$

\begin{tabular}{lccc}
\hline Exposure duration & $24 \mathrm{~h}$ & $48 \mathrm{~h}$ & $72 \mathrm{~h}$ \\
\hline $\mathrm{U}$ depl. & $136(115-172)$ & $134(126-143)$ & $157(148-161)$ \\
${ }^{233} \mathrm{U}$ & $131(120-145)$ & $151(139-159)$ & $231(208-282)$
\end{tabular}




\subsection{Subcellular effects}

After 24 hrs of exposure we could observe that algal growth were roughly the same for the contaminated and the reference cultures. As shown in table 3, algal density increased from 441559 cell. $\mathrm{mL}^{-1}$ to 485 000 cell. $\mathrm{mL}^{-1}$ which corresponds to $10 \%$ algal growth. On the other hand, algal biovolume was doubled between the beginning and the end of the experiment. Thus, biovolume seemed to be more sensible parameters to normalize the results from the other analyses.

Table 3. Growth parameters of C. reinhardtii exposed to U depl. and ${ }^{233} \mathrm{U}$ during $24 \mathrm{hrs}$.

\begin{tabular}{llllll|lllll}
\multicolumn{8}{c}{ Cellular density $\left(\right.$ cell. $\left.\mathrm{mL}^{-1}\right)$} & \multicolumn{4}{c}{ Total algal biovolume $\left(\mathrm{mm}^{3}\right)$} \\
\cline { 2 - 11 } & $0 \mathrm{~h}$ & $3 \mathrm{~h}$ & $6 \mathrm{~h}$ & $12 \mathrm{~h}$ & $24 \mathrm{~h}$ & $0 \mathrm{~h}$ & $3 \mathrm{~h}$ & $6 \mathrm{~h}$ & $12 \mathrm{~h}$ & $24 \mathrm{~h}$ \\
\hline Control & 441559 & 438067 & 425733 & 471733 & 489533 & 2.29 & 2.47 & 2.67 & 4.43 & 4.80 \\
U depl. & 441559 & 439133 & 459600 & 507667 & 480600 & 2.29 & 2.44 & 3.12 & 4.14 & 4.43 \\
${ }^{233} \mathrm{U}$ & 441559 & 441667 & 457867 & 511333 & 484267 & 2.29 & 2.51 & 2.96 & 3.96 & 5.12
\end{tabular}

Fluorescence emitted in the presence of DCMU is the same for each treatment and exposure time (table 4). Without DCMU the fluorescence emitted by the contaminated algae is higher than that of control algae after $24 \mathrm{hrs}$ of exposure.

Table 4. Chlorophyll fluorescence measurement $\left(\lambda_{\text {excitation }}=450 \mathrm{~nm}, \lambda_{\text {emission }}=680 \mathrm{~nm}\right)\left(\mathrm{AFU} / \mathrm{mm}^{3}\right.$ algae$)$.

\begin{tabular}{cccccc|ccccc} 
& \multicolumn{4}{c|}{ With DCMU } & \multicolumn{5}{c}{ Without DCMU } \\
\cline { 2 - 11 } Time (h) & $0 \mathrm{~h}$ & $3 \mathrm{~h}$ & $6 \mathrm{~h}$ & $12 \mathrm{~h}$ & $24 \mathrm{~h}$ & $0 \mathrm{~h}$ & $3 \mathrm{~h}$ & $6 \mathrm{~h}$ & $12 \mathrm{~h}$ & $24 \mathrm{~h}$ \\
\hline Control & $36.0 \pm 2.2$ & $32.9 \pm 5.3$ & $34.6 \pm 0.7$ & $35.6 \pm 0.1$ & $29.4 \pm 2.3$ & $15.8 \pm 2.1$ & $14.6 \pm 0.9$ & $17.7 \pm 2.1$ & $14.4 \pm 0.1$ & $13.6 \pm 0.4$ \\
U depl. & $36.0 \pm 2.2$ & $37.4 \pm 3.4$ & $33.3 \pm 2.7$ & $37.5 \pm 0.8$ & $33.6 \pm 0.7$ & $15.8 \pm 2.1$ & $15.2 \pm 1.4$ & $19.0 \pm 1.9$ & $15.5 \pm 0.5$ & $21.4 \pm 2.6$ \\
${ }^{233} \mathrm{U}$ & $36.0 \pm 2.2$ & $33.3 \pm 2.7$ & $32.6 \pm 0.7$ & $38.9 \pm 1.6$ & $28.9 \pm 1.7$ & $15.8 \pm 2.1$ & $14.2 \pm 0.4$ & $20.3 \pm 0.6$ & $14.2 \pm 0.3$ & $19.2 \pm 0.6$
\end{tabular}

Thiol compounds analysis by HPLC was optimised using C. reinhardtii algae contaminated by $10 \mu \mathrm{M}$ of cadmium during $96 \mathrm{hrs}$. As shown in figures 1a and 1b, our experimental procedure allows to separate and to identify different thiol compounds.
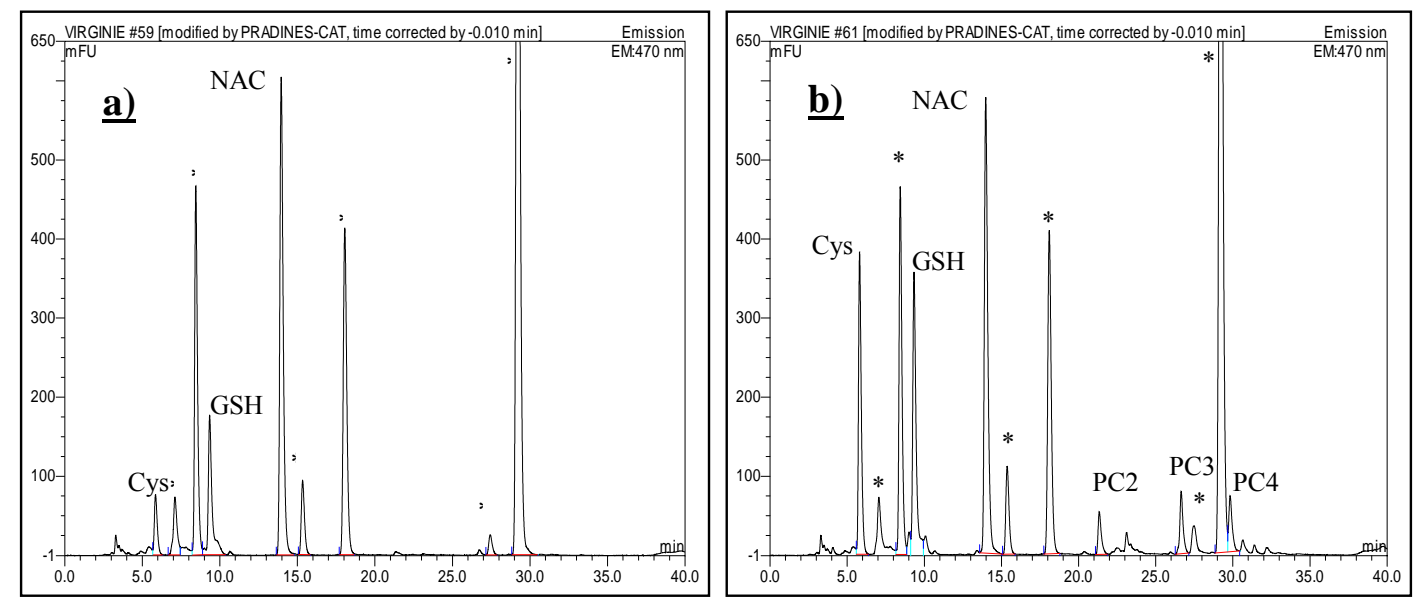

Figure 1. Chromatograms of thiol compounds in C. reinhardtii. a) $10 \mu \mathrm{M} \mathrm{Cd}$; b) control (* reagent).

Retention time of each non-protein thiol was checked using different mBBr-labeled standards. Cysteine and reduced GSH were the major thiol present in the cytosol of C. reinhardtii. As expected, cadmium contamination induced the production of phytochelatins 2,3 and 4 and modified cysteine 
and reduced GSH levels. Nevertheless total phytochelatins-SH level was lower than that of reduced GSH and cysteine. HPLC analysis of uranium contaminated C. reinhardtii did not allow to observe phytochelatins induction for any exposure times. This result was observed in the same manner for $\mathrm{U}$ depl. and for ${ }^{233} \mathrm{U}$ (data not shown).

Table 5 presents results of GSH/[GSH + 0.5 GSSG] ratio calculated from HPLC analysis. Total GSH levels were obtained by pre-column reduction step using TCEP. The ratio is relatively weak $(\sim 0.6)$ and decreased during the experiment period to reach about 0.4 after $24 \mathrm{hrs}$ of exposure in presence or absence of uranium.

Table 5. Glutathione redox ratio of C. reinhardtii during $U$ depl. or ${ }^{233} \mathrm{U}$ contamination assay (no duplicate run).

\begin{tabular}{c|c|c|c|c|c}
\multicolumn{2}{l|}{$0 \mathrm{~h}$} & $3 \mathrm{~h}$ & $6 \mathrm{~h}$ & $12 \mathrm{~h}$ & $24 \mathrm{~h}$ \\
\hline Control & 0.588 & 0.737 & 0.634 & 0.55 & 0.4 \\
U depl. & 0.588 & 0.466 & 0.574 & 0.473 & 0.381 \\
${ }_{233} \mathrm{U}$ & 0.588 & 0.511 & 0.519 & 0.558 & 0.383
\end{tabular}

\section{DISCUSSION}

The objectives of this work were to identify pertinent biomarkers of uranium effects on the microalga C. reinhardtii. First experiments on chronic (24-72 h) growth inhibition showed that no difference between $U$ depl. and ${ }^{233} U$ could be observed and that $E_{50} \mathrm{~s}$ were c.a. 150 nmol U.L ${ }^{-1}$. Despite enhanced delivered radiological doses by ${ }^{233} \mathrm{U}$, (a factor of 10.000 compared to $U$ depl.), the dose rates per cell remains low (c.a. $10 \mathrm{mGy} /$ day - calculation not shown), that could explain this absence of difference. In order to test different parameters, C. reinhardtii algae were contaminated with $U$ depl. (mainly chemotoxic) and ${ }^{233} \mathrm{U}$ (chemo- and possibly radiotoxic) during $24 \mathrm{hrs}$. Several samplings were realised at regular time intervals. Algae cultures were contaminated with $150 \mathrm{nM}$ uranium corresponding to EC50. In these conditions, algae grew similarly in each condition. We observed only $10 \%$ increased of algal density at the end of the assay indicating that algae did not divide. Nevertheless, cell ultra-structure seemed to be affected by the experimental conditions since algal biovolume was doubled. This observation could be explain by the concentration of algae at the beginning of the experiment which is 176 times higher in this experiment (c.a. 500000 cell. $\mathrm{mL}^{-1}$ ) than in the inhibition concentration experiment $\left(2500\right.$ cell. $\left.\mathrm{mL}^{-1}\right)$. In this condition algae were probably deprived. Furthermore, centrifugation protocol (18 $500 \mathrm{x} \mathrm{g}, 15 \mathrm{~min})$ used to prepare and to collect algae for contamination experiment and biomarkers analysis may induce stress. This hypothesis was supported by the glutathione redox ratio analysis. At the beginning of the experiment this ratio is about 0.6 suggesting that the $C$. reinhardtii cells were already stressed before the contamination and it decreased during the exposure probably because of nutrient deprivation. No significant difference was observed between control and contaminated cells. The low values of glutathione redox ratio may mask a potential effect of uranium. Microalgae are known to detoxify metal by synthesis of metal-binding proteins such as phytochelatins. In order to observe phytochelatin production in C. reinhardtii we first exposed the algae to $10 \mu \mathrm{M}$ of Cadmium. This metal is known to promote oxidative damage by reducing the cellular antioxidant capacity [10] and it is also one of the most effective activator of phytochelatin biosynthesis [6]. HPLC analysis shows that cadmium induced production of phytochelatins 2,3 and 4 with a major proportion of PC3. In presence of $150 \mathrm{nM}$ uranium, no phytochelatin was detected. This signifies that uranium and/or ionising radiation would not be able to promote phytochelatin biosynthesis in our experimental conditions or that phytochelatin production was lower than the detection limit. Fluorescence measurement is a parameter, which is an indication of physiological status of the cells. It refers to photosystem II (PSII) activity. Inhibition of PSII by DCMU allow to measure maximum fluorescence. Whitout DCMU treatment the fluorescence emitted is inversely-proportional to PSII activity. Total fluorescence levels were approximately the same for each treatment and were stable during all the experiment. In absence of DCMU, no effect could be observed after $12 \mathrm{hrs}$ of exposure. Fluorescence measured on samples collected after $24 \mathrm{hrs}$ were lower for control than for uranium-contaminated cells. $\mathrm{U}$ depl. and ${ }^{233} \mathrm{U}$ fluorescence values were nearly the same and correspond to about $65 \%$ of the total fluorescence at the same time, whereas $46 \%$ 
of total fluorescence was measured for the control. This results show that uranium induced an inhibition of PSII activity after $24 \mathrm{hrs}$ of exposure. This inhibition was similar for the two isotopes tested indicating that this effect was related to the chemotoxicity of the element. Thus, PSII activity seems to be a good indicator of early toxic effect after uranium exposure.

\section{CONCLUSIONS}

This work underlined the interest to measure several biomarkers, related to different metabolic activities, to observed the toxicity of an element on a given organisms. Glutathione redox ratio seemed to be disturbed by the experimental conditions, which had probably induced a stress before the beginning of the contamination. Consequently, experimental procedure will be optimised and other oxidative stress biomarkers such as enzymatic activities (superoxide dismutase, catalase ascorbate peroxidase) will be investigated. No phytochelatin was detected after uranium exposure in our experimental conditions. On the other hand, fluorescence measurement proved to be a good indicator of earlier toxic effects induced by uranium contamination on C. reinhardtii. The study of this parameter will be completed by the Pulse-Amplitude-Modulation (PAM) method in order to assess quantitatively the photosynthetic activity. Finally, no difference was observed between $U$ depl. and ${ }^{233} U$ exposure during the growth rate inhibition assay and the biochemical analysis signifying that uranium is essentially chemotoxic. This result could be explained by the low dose rate delivered by $150 \mathrm{nM}^{233} \mathrm{U}$.

\section{Acknowledgments}

The authors are grateful to M. Morello for ${ }^{233} \mathrm{U}$ analysis and to S. Cuine (CEA, Cadarache) for giving synthetic phytochelatins. This work was supported by ENVIRHOM program of IRSN.

\section{References}

[1] Colle C. et al., Uranium (In Métivier H., ed. L'Uranium de L'environnement à l'Homme. EDP Sciences, Paris, France, 2001) 187-211.

[2] Franklin N.M et al., Aquat. Tox. 48 (2000) 275-289.

[3] Charles A.L. et al., Aquat. Tox. 60 (2002) 61-73.

[4] Fortin C. et al., Environ. Toxicol. Chem. 23 (2004) 974-981.

[5] Pinto E., J. Physiol. 39 (2003) 1008-1018.

[6] Ahner B., Morel F. Prog. Phycol. Res. 13 (1999) 1-31.

[7] Fortin C., Campbell P.G.C., Environ. Toxicol. Chem. 19 (2000) 2769-2778.

[8] Sueoka N, et al., J. Mol Biol. 25 (1967) 47-66.

[9] Rijstenbil J.W., Wijnholds J.A, Mar. Biol. 127 (1996) 45-54.

[10] Sies H. Free Radic. Biol. Med. 27 (1999) 916-921. 